JOURNAL OF APPLIED CRYSTALLOGRAPHY

ISSN 1600-5767

\section{Handbook of Carbon Nano Materials Volume 7, Synthetic Developments of Graphene and Nano- tubes. Edited by Francis D'Souza and Karl M. Kadish. World Scientific Press, 2016. Pp. 240. Price GBP 195.00, USD 295.00 (for the Volume 7 and 8 set). ISBN 9789814689175.}

\author{
Manuel Dossot*
}

LCMPE UMR 7564, CNRS/Université de Lorraine, 405 rue de Vandoeuvre, 54601 Villers-lès-Nancy, France. *Correspondence e-mail: manuel.dossot@univ-lorraine.fr

Volume 7 of the Handbook of Carbon Nano Materials is devoted to the vast topic of synthesis, modification and applications of $s p^{2}$ carbon allotropes, mainly graphene and carbon nanotubes (CNTs). The book is divided into five chapters aimed at giving a general overview of graphene and CNT synthesis, chemistry and applications, with some emphasis put on more specific subjects. For instance, the reader will discover some original methods in the context of CNT analysis, such as surface enhanced Raman spectroscopy, and will be introduced to the effects on CNT electronic properties of filling them with nanoparticles. This book belongs to a series entitled Handbook of Carbon Nano Materials, and therefore the reader could expect that the contents of Volume 7 will be up to date and self-consistent. The cover claims that this book should 'provide a comprehensive overview of the author's work and significant discoveries and pioneering contributions from other groups' and that it is a 'highly useful book for graduate students, as well as beginning and senior researchers'. Unfortunately, both claims are far from being fulfilled for several reasons.

Reviewing a vast topic like synthesis, chemistry and applications of graphene and CNTs requires being clear, concise, up to date, pertinent and original if compared to the excellent textbooks already available (to cite only two of them, Carbon Nanotubes and Graphene, 2nd ed., edited by K. Tanaka and S. Iijima, and Graphene: Synthesis and Applications, edited by W. Choi and J. W. Lee). There are also excellent reviews on these topics in the literature, written by specialists in their respective areas, and it is therefore a difficult task to produce a new book with original points of view. One would at least expect to read a pedagogical overview of the subject and a well organized analysis of carefully selected references (among the $\sim 100000$ currently available in the chemical literature). This is not the case with the present book. Several flaws blur the scientific message, in particular the lack of logical organization of the chapters.

Chapter 1 is devoted to graphene synthesis and applications. It first gives some general properties of graphene, but without going into details. The structure of graphene is briefly explained, and on page 3 the reader encounters a first, very enthusiastic sentence: it is said that 'graphene is the mother of all other allotropes of carbon'. People working, for instance, with the diamond forms of carbon may disagree with this kind of sentence. The mechanical, electrical, thermal and optical properties of graphene are briefly presented from only an experimental point of view, and while some allusions are made to theoretical work, it is not further developed anywhere in this book. Graphene synthetic routes are very briefly provided, but no idea about growth mechanisms is furnished, nor why people use specific conditions. This part merely consists of an enumeration of synthetic methods, sometimes giving very precise experimental details extracted from the experimental methods of the cited references, but with no rationale of the growth process. Graphene applications are overviewed very quickly and the very abundant literature is not adequately represented.

In fact, a better introduction to graphene properties and synthesis can be found in Chapter 4. This chapter, dealing with the chemical vapor deposition (CVD) synthetic processes of carbon nanomaterials, presents a very clear and nicely written introduction 
to graphene synthesis. In fact, Chapter 4 in my opinion is the most valuable chapter of this book, clearly written, organized, with a list of valuable references and a good choice of figures to illustrate the text. The authors of this chapter make the effort to recall the recommended nomenclature for twodimensional graphene samples and give a very pedagogical introduction to graphene's physicochemical properties, useful for anyone interested in working on the subject. The book's lack of logical organization is also obvious when reading Chapters 2 and 5. Chapter 2 deals with the 'synthesis and characterization of carbon nanotubes', and Chapter 5 is entitled 'single-walled carbon nanotubes: synthesis and modification of the electronic structures'. Some pieces of information in these chapters are redundant, and it would have been more logical to make these chapters consecutive.

Another point is the number of typographical errors contained in the text. It would be too fastidious to give here the complete list, but I illustrate this by giving one example on pages 118-119. Figure 19 in Chapter 3 deals with nitrogendoped graphene, while the text on page 118 mentions a reference dealing with nitrogen-doped carbon nanofibres. The same mistake is made with Figure 20, which does not correspond to the text on page 118 . This does not help the reader, especially students who may be confused by such errors.

Chapter 2 furnishes a brief overview of the CVD synthetic methods for producing carbon nanotubes and develops in more detail the electrochemical technique. This part is interesting, as well as the report of characterization techniques at the nanometre scale of CNTs that follows. Though it is focused on some specific techniques such as surface-enhanced Raman scattering measurements, it is preceded by a good presentation of some generalities on resonant Raman spectroscopy of CNTs, which is in fact lacking in Chapter 5.

Chapter 3 reports some results on the synthesis and modification of mesoporous carbon structures, nanocomposites with graphene oxide or reduced graphene particles, and carbon nanofibres. Though interesting, and clearly written, the relationship to the rest of the book becomes evident only when the authors give some results on boron- or nitrogendoped graphene samples. This is always the kind of trap one may fall into when dealing with carbon allotropes: there are so many carbon (nano)materials that, if one does not restrict oneself to specific materials, a whole book could be written with a succession of chapters that might be simply juxtaposed rather than being adequately organized. This is indeed the main flaw of the present book.

Finally, Chapter 5 would have been a good review of singlewalled CNT synthesis if the references concerning this subject included those after 2006. It would seem that nothing has happened in the subject in the past decade when one reads this chapter, which is far from being true. Chirality-specific synthesis has made serious breakthroughs using, for instance, CVD processes over the past five years, and nothing is said in the text of this chapter. No information is given on the relationship between the catalyst surface properties and the diameter and chirality of the grown CNTs; there is no deep physicochemical discussion about the role of gas pressure, growth temperature or the chemical nature of the substrate. The second part of this chapter deals with the control of electronic properties of single-walled CNTs by filling them with various particles. This is interesting and clearly written, but it also lacks a consideration of water-filled CNTs, which is clearly a very important topic in view of sensing applications in a water medium of CNT devices.

Considering these flaws, the goal of this book is far from being reached. Students will find little of interest since the presentation is sometimes confusing or material is not organized in a logical way, and better textbooks are available on the subject. Researchers can pick up some useful information in some chapters, principally if they are interested in experimental details on synthetic methods for graphene or CNTs, but the text sometimes lacks references to recent literature and is not self-sufficient. I therefore do not consider this book as a 'must have' on bookshelves. 